\title{
Capital social, confianza y modelos de asociacionismo en España
}

\author{
José Atilano Pena-López \\ José Manuel Sánchez-Santos \\ Universidade da Coruña. Facultad de Economía y Empresa. Departamento de Economía \\ atilano.pena@udc.es; jose.manuel.sanchez@udc.es
}

Recepción: 11-11-2016

Aceptación: 27-04-2017

\section{Resumen}

El presente trabajo estudia la estructura del asociacionismo en España con el objeto de contrastar su incidencia sobre la confianza generalizada. Con este objetivo se analizan, a partir de los datos de una encuesta sobre pertenencia asociativa realizada en España, el vínculo existente entre las redes de pertenencia asociativa de cada individuo y la confianza generalizada. Junto a la existencia de una evolución de la estructura asociativa hacia un predominio de formas más horizontales, los resultados ponen de relieve la influencia de las pertenencias múltiples en la generación de confianza generalizada, tal y como predice la hipótesis de Paxton (2007). En conjunto, tanto la integración en asociaciones abiertas como la influencia de factores personales ligados a la función de bienestar psicológico (Uslaner, 2002) han resultado significativos en la formación de la confianza generalizada.

Palabras clave: confianza generalizada; capital social; capital social individual; asociacionismo en España

\begin{abstract}
Social capital, trust and models of associationism in Spain
This paper studies the structure of associations in Spain in order to examine its influence on generalized trust. Using data from a survey conducted in Spain on associative belonging, the link between individuals' networks of associational membership and generalized trust is analyzed. The results highlight that the associative structure in Spain has evolved towards more horizontal forms and that multiple belongings play a role in generating generalized trust, as predicted by Paxton's hypothesis (2007). Both integration in open associations and personal factors linked to the role of psychological well-being (Uslaner, 2002) have proved significant in the formation of generalized trust.
\end{abstract}

Keywords: generalized trust; social capital; individual social capital; associations in Spain 


\section{Sumario}

1. Capital social y confianza generalizada

2. Confianza generalizada $\mathrm{y}$ asociacionismo
3. Estructura del asociacionismo en España y confianza generalizada. Evidencia empírica

4. Conclusiones

Referencias bibliográficas

\section{Capital social y confianza generalizada}

El éxito del concepto de capital social está asociado en gran medida a su presunta capacidad para situar el hecho económico dentro del entramado social, es decir, permite abordar la cuestión de la embeddedness o imbricación de lo económico en lo social ${ }^{1}$. En esta línea, son numerosos los trabajos que estudian la influencia del capital social sobre variables tan relevantes como el crecimiento económico, la distribución de la renta, la calidad de la vida en general e incluso sobre la evolución de la estructura y de la identidad social (Dasgupta y Serageldin, 2001; Woolcock, 2001).

La investigación de capital social ofrece un complejo entramado conceptual que permite integrar diversas corrientes de investigación procedentes de la sociología, de la ciencia política y de la economía (Adler y Kwon, 2002; Sobel, 2002). Esta aproximación interdisciplinar da lugar a una cierta falta de consenso básico (Millán y Gordon, 2004)². En una definición de síntesis, se trata de una forma de capital colectivo producto de un proceso de inversión individual y social de recursos y de tiempo materializado en redes y en normas que facilitan el funcionamiento social y cuantificado habitualmente

1. Obviamente, no es la única orientación que aborda este problema, aunque sí una de las de mayor progresión reciente. Junto a ella están la sociología de la economía, de raíz weberiana (Granovetter, 1973; Etzioni, 2007) y la economía institucional (Williamson, 2000). Para la primera, desde el punto de vista sociológico, es obvio que la acción económica no puede separarse de la búsqueda de aprobación, estatus, sociabilidad y poder, en tanto que, para la segunda, las instituciones y las redes de relaciones generadas son simples modos de reducción de costes de transacción e información.

2. La definición de Coleman (1988) y la interpretación crítica de Bourdieu (2001) adoptan una perspectiva individual o micro que considera que el capital social se refiere a aspectos de la estructura social, obligaciones y expectativas, canales de información, conjunto de normas y sistemas de sanción que condicionan los comportamientos individuales. Bourdieu, en clave marxista, pero sin abandonar este enfoque individual, plantea que el capital social es un instrumento para que individuos y grupos reproduzcan sus ventajas sociales mediante el dominio de redes de influencia. Putnam (1995), en cambio, opta por centrarse en aspectos sociales generales o "culturales», es decir, adopta un enfoque centrado en lo macrosocial o en rasgos de la sociedad en conjunto. Así, su visión del capital social se centra en la generación de comportamientos prosociales o que generan comunidad: rasgos o características de las organizaciones sociales como normas, redes y confianza que facilitan la cooperación y la coordinación para el beneficio mutuo. Las definiciones manejadas en la mayor parte de los estudios priman lo colectivo sobre los individual (Bowles y Gintis, 2002; Paldam, 2000; Winter, 2001). 
mediante la variable confianza generalizada. Por un lado, el capital social es propiedad del individuo, que se materializa en su agenda personal, de ahí la denominación en el mundo anglosajón de rolodex $x^{3}$ capital, que este emplea para extraer recursos. Por otro lado, el capital social es una propiedad del grupo que tiene rasgos de bien público, ya que todos los miembros perciben externalidades (positivas o negativas ${ }^{4}$ ) de esa red con la que están vinculados directa o indirectamente.

La corriente principal en la teoría del capital social supone la existencia de un vínculo directo entre confianza y participación asociativa, evaluadas ambas a nivel macro (Putnam, 1995, 2000). No obstante, dicha identidad entre confianza, redes asociativas y redes individuales dista de ser evidente (Morales y Mota, 2006: 102). De hecho, la denominada "controversia Olson-Putnam» (Pena y Sánchez, 2005, 2007) se centra precisamente en la ambigua relación entre ambas variables.

En un intento de profundizar en el estudio de esta cuestión, el objetivo del presente trabajo es estudiar la vinculación entre la confianza generalizada (proxy del capital social) y las redes asociativas que plantea la teoría del capital social. Para ello, adoptamos una perspectiva micro y analizamos el modo en que la extensión, la estructura y las características de la pertenencia asociativa de un individuo influye en que este manifieste o no confianza respecto a su comunidad de referencia. Siguiendo la perspectiva de Paxton (2007), buscamos contrastar en qué medida este vínculo es sólido en todos los casos o únicamente producto de un determinado tipo de asociaciones o de una particular configuración de las pertenencias asociativas.

El resto del trabajo se estructura como sigue. En el segundo apartado nos centramos en la teoría del capital social como base de nuestro análisis, en concreto, el estudio de la interrelación entre redes asociativas y confianza generalizada. En el tercero estudiamos la estructuración de las redes asociativas personales en España para, seguidamente, sobre la base de una encuesta, realizar un contraste de la interrelación entre las pertenencias asociativas, en particular el efecto de las pertenencias múltiples que propone Paxton y la confianza generalizada. Finalmente se exponen las principales conclusiones.

\section{Confianza generalizada y asociacionismo}

La confianza generalizada o despersonalizada es la percepción de que la generalidad de la sociedad es confiable. Se trata de un conjunto de expectativas aprendidas o confirmadas socialmente sobre el comportamiento de los otros, las organizaciones y las instituciones que lleva a los individuos a afirmar que la

3. Esta palabra alude a las populares agendas de teléfono de empresa compuestas por fichas dispuestas en un eje giratorio. Así, la mayor inversión en capital social de un individuo sería equiparable a una mayor agenda personal.

4. No todos los efectos del capital social tienen que ser necesariamente positivos. La corrupción puede ser considerada también una expresión de capital social (Pena y Sánchez, 2014). 
mayoría de los integrantes de una comunidad determinada es confiable (Herreros, 2003, 2008; Barber, 1983; Luhmann, 1988). Esta forma de confianza inespecífica, no estratégica y basada en una visión «benigna» de la sociedad proporciona, entre otras cosas, una disminución de los costes de transacción y una mayor capacidad de asociación con otros individuos provenientes de grupos ajenos (bridge) (Uslaner, 2002, 2008).

Las propuestas explicativas del desarrollo de la confianza generalizada parten de la confianza como un constructo derivado de las interacciones (Hardin, 2001). Los individuos confían en los otros en la medida en que mantienen un vínculo con ellos. No obstante, la confianza en el otro, más allá de grupos particularistas, no es inmediata. Esta podría provenir de un conocimiento inferido de los mismos a partir de las normas que esperamos que el otro cumpla (Hearn, 1997). Así, la homogeneidad social en normas, valores, intereses e identidades favorece la confianza, y la diversidad la perjudica (Hardin, 2001). Al mismo tiempo, la frecuencia de las interacciones eleva los niveles de confianza en las mismas e incrementa la confianza general (Sorrentino et al., 1995), al facilitar la comprensión de las señales enviadas por los demás (Bacharach y Gambeta, $2001)^{5}$. Del mismo modo, algunos autores sostienen que cuanto mayor sea la pertenencia de un individuo a algunas asociaciones, mayor tiende a ser su nivel de confianza general (Stolle, 2001).

Simplificando el entramado teórico, podemos dividir las propuestas explicativas sobre la confianza generalizada en dos grandes grupos en razón de la variable priorizada como determinante de dicha confianza. Por una parte, están aquellas posturas que enfatizan la existencia de una predisposición psicológica alterable por las experiencias cotidianas (Uslaner, 2002, 2008) y, por otra, aquellas que defienden que se deriva de un aprendizaje social (Paxton, 2007). La aparente contraposición entre ambas propuestas es solo parcial, dado que la causalidad de la confianza generalizada es múltiple y depende tanto de factores individuales como sociales.

Dentro del primer grupo de propuestas se encuadran tanto aquellos que consideran que la confianza generalizada tiene una base innata como aquellos que defienden que es aprendida en un momento concreto del proceso de desarrollo. En ambas posturas se defiende que las decisiones actuales están arraigadas en un aprendizaje previo frente al cual las experiencias actuales serían menos relevantes (Uslaner, 1999; Becker, 1996; Jones, 1996). No obstante, conviene puntualizar lo señalado por algunos autores que participan de esta perspectiva. Uslaner $(2002,2008)$ considera que la confianza es una función del bienestar psicológico y, en particular, el sentido de control sobre la propia vida es la base de comprensión de esta variable. En esta misma línea, otros estudios han evidenciado el peso que las variables de carácter estructural pueden tener en los niveles de confianza generalizada, bien del entorno inmediato o vecindario (heterogeneidad étnica del vecindario, seguridad percibida, etc.),

5. Los encuentros virtuales se han revelado como notablemente menos eficaces (Ostrom y Walker, 2003). 
bien de las características nacionales (renta, diversidad cultural, esperanza de vida, etc.) (Delhey y Newton, 2005; Delhey et al., 2011). En suma, la confianza es un producto de la socialización del individuo en el que es crucial que este disponga de una red social personal de apoyo lo suficientemente sólida que garantice un bienestar subjetivo y un control sobre su entorno.

El enfoque centrado en el aprendizaje social, en cambio, sugiere que los individuos extrapolan de forma continua sus experiencias personales reiteradas a sus estimaciones generales (Hardin, 2001; Yosano y Hayashi, 2005). La confianza generalizada sería, así, el resultado de la suma de las experiencias individuales en los diversos dominios vitales (Glanville y Paxton, 2007; Burns y Kinder, 2000). Desde esta perspectiva, la pertenencia a asociaciones podría justificar un mayor nivel de confianza generalizada, puesto que las asociaciones multiplican las posibilidades de interacción. Dado que las normas se difunden a través de los grupos influenciando actitudes y conductas, la confianza arraigada en valores compartidos, cooperación y comportamientos predecibles es más probable que se difunda mediante el asociacionismo (Zucker, 1986; Marsden y Friedkin, 1993). En un sentido más amplio, la mera participación política ya genera este efecto, pero este se ve potenciado mediante la actividad asociativa (Morales, 2006). Por el contrario, aquellos que disponen de experiencias sociales más limitadas presentan una baja capacidad de predicción de comportamientos de aquellos ajenos a su propio grupo.

En definitiva, el que confía en los miembros de una determinada red asociativa adquiere la práctica de la confianza generalizada (Coleman, 1990). Ahora bien, este salto no es del todo inmediato, porque los mecanismos que generan confianza en grupos particulares no tienen por qué ser necesariamente efectivos a escala macrosocial (Stolle y Rochon, 1998). En este ámbito, la hipótesis sostenida por Paxton (2007) establece que este proceso de extensión se deriva de las interconexiones que existen entre las propias asociaciones. Por lo tanto, de acuerdo con esta hipótesis, sería la propia vertebración del asociacionismo - en particular, las pertenencias múltiples- la que permitiría una extensión de la percepción de confianza generalizada.

Un individuo que conecta dos asociaciones facilita la interconexión con una comunidad más grande y la transición de la confianza particular interna a una asociación a la confianza general. Así, en tanto que una cadena de asociaciones cerradas, facilita un número muy limitado de interconexiones entre individuos, la interconexión de asociaciones las multiplica, lo que facilita la generalización de valores y normas, así como la confianza general ${ }^{6}$ (véase la figura 1). Este argumento nos lleva a la distinción entre asociacionismo abierto y asociacionismo cerrado. El primero facilita la integración en un amplio

6. Interpretado desde la perspectiva de la psicología social, los individuos categorizan a los otros en dos grupos: nosotros y ellos (Hogg, 1992). Aquellos que se integran en el nosotros son percibidos en términos de confianza. Así, un individuo que se integra en diversos grupos tiende a generalizar la concepción del nosotros $\mathrm{y}$, consecuentemente, a incrementar su percepción de confianza (Herreros, 2003). 
Figura 1. Asociacionismo cerrado frente a asociacionismo abierto
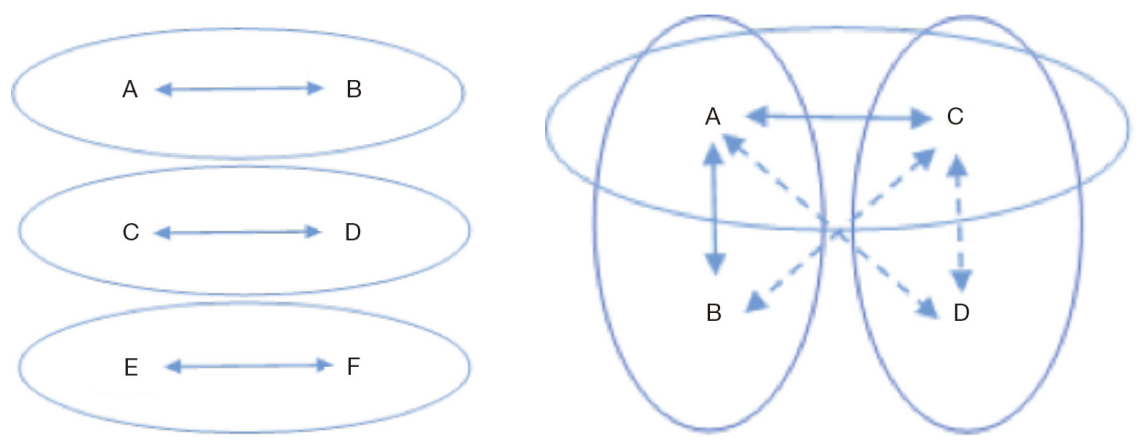

Fuente: elaboración propia a partir de Paxton (2007).

entramado asociativo de naturaleza diversa y resulta beneficioso para la confianza general. El segundo, por el contrario, dificulta la integración en otras formas de asociacionismo y carece de efectos sobre la confianza generalizada. Una hipótesis análoga puede encontrarse en Herreros (2002). De acuerdo con este autor, la generalización de la confianza se debe a la aproximación o al amoldamiento de las preferencias derivado de las interacciones propias de la actividad asociativa y al efecto de extensión de los mismos derivados de los múltiples contactos ligados al asociacionismo. Ambos condicionantes dependen de la naturaleza de la organización. Es decir, la confianza generalizada no puede ligarse exclusivamente a la pertenencia a cualquier forma asociativa, sino solo a aquellas consideradas más abiertas ${ }^{7}$.

De este modo, la interconexión entre asociaciones originada por las pertenencias múltiples (Moody y White, 2003) permiten realizar transferencia de confianza entre entidades asociativas e incrementar la confianza generalizada. Consecuentemente, la confianza generalizada no se asienta únicamente en la multiplicidad de asociaciones y en la diversidad dentro de las mismas, como se derivaría directamente de las propuestas de Putnam (2000) o Granovetter (1973). En realidad, dicha confianza aparece más vinculada a la diversidad de grupos, a la diversidad dentro de los grupos y a las interconexiones en esa diversidad de grupos.

Llegados a este punto, se puede concretar la hipótesis de Paxton (2002, 2007) en los siguientes términos: una mayor incorporación de individuos a asociaciones interconectadas o abiertas tendrá un efecto positivo en la confianza generalizada y, por tanto, en la generación de capital social. Por el contrario, la pertenencia a asociaciones aisladas carece de efecto en términos de confianza generalizada.

7. Esta distinción está ligada a otras distinciones comunes en los estudios de capital social, los lazos débiles de Granovetter y la distinción entre bonding y linking planteada por Putnam (2000), pero se centra más en la naturaleza de las asociaciones que en la diversidad interna de dichas asociaciones. 
La contrastación de esta propuesta explicativa exige controlar simultáneamente aquellas variables contextuales (satisfacción vital, nivel de ingresos y estudios, etc.) que pueden resultar un apoyo a las propuestas explicativas que señalan que tanto el entorno social de pertenencia como el bienestar subjetivo y material son determinantes decisivos en la confianza generalizada. A este respecto, es importante señalar que solo se trata de una aparente oposición entre ambas propuestas explicativas. Los niveles de asociacionismo guardan una relación igualmente con el bienestar subjetivo o la homogeneidad del entorno. La contrastación de esta hipótesis se enfrenta a un problema de endogeneidad que ha sido reiteradamente abordado por la literatura con evidencias contrapuestas. Teóricamente es posible argumentar tanto que la confianza genera una mayor propensión al asociacionismo como que el asociacionismo favorece la confianza generalizada. No obstante, la mayor parte de la evidencia empírica disponible ha puesto de relieve una vinculación causal unidireccional del asociacionismo hacia la confianza (Claibourn y Martin, 2000). En sentido contrario, merece la pena destacar los trabajos de Sonderskov (2011) y el más reciente de Ingen y Bekkers (2015). Este último llega a conclusiones muy relevantes sobre los efectos del asociacionismo, que, en cierta medida, disuelven la aporía. Aunque no hay evidencias concluyentes de que la causalidad vaya del asociacionismo a la confianza, el asociacionismo aglutina a aquellos individuos que presentan mayores niveles de confianza, de forma que se constituye como un pool of democracy con carácter bidireccional. No obstante, tal y como hemos señalado previamente, nuestro trabajo no se centrará en este problema de endogeneidad, sino en el carácter diferencial de las diversas formas de asociacionismo y, en particular, de su entrelazado y los vínculos existentes con la confianza generalizada. En última instancia, se trata de delimitar en qué contextos se está creando la confianza generalizada.

\section{Estructura del asociacionismo en España y confianza generalizada. Evidencia empírica}

La contrastación empírica de lo que hemos denominado "hipótesis Paxton» requiere una medición del nivel de pertenencia asociativa y, simultáneamente, el estudio de las interrelaciones entre las pertenencias a múltiples asociaciones. Además, también exige estudiar los niveles individuales de confianza general controlando el conjunto de variables sociodemográficas.

\subsection{Pertenencia y múltiples pertenencias asociativas}

La base de nuestra contrastación empírica es la encuesta sobre capital social y desigualdad en España, desarrollada por el grupo OSIM en 2011 $1^{8}$. En ella se

8. La encuesta sobre capital social y desigualdad en España fue realizada en 2011 sobre un universo de personas mayores de 18 años residentes en España. El tamaño de la muestra fue de 3400 entrevistas individuales, y el error muestral de $\pm 1,7 \%$ para datos globales, para un 
Tabla 1. Pregunta sobre pertenencia a asociaciones

P7. Voy a leerle una lista de organizaciones voluntarias. Dígame, por favor, si es Vd. miembro de alguna de ellas. (Codificar cada respuesta negativa con un 1. Si es afirmativa, se pregunta si participa activamente, en cuyo caso se codifica con un 3 , y si no es el caso, con un 2.)

\begin{tabular}{|c|c|c|c|c|}
\hline & & $\begin{array}{c}\text { No } \\
\text { pertenece }\end{array}$ & Pertenece & $\begin{array}{l}\text { Participa } \\
\text { activamente }\end{array}$ \\
\hline V34 & Organizaciones religiosas & 1 & 2 & 3 \\
\hline V35 & Organizaciones deportivas & 1 & 2 & 3 \\
\hline V36 & $\begin{array}{l}\text { Organizaciones educativas, artísticas, musicales } \\
\text { o culturales }\end{array}$ & 1 & 2 & 3 \\
\hline V37 & Sindicatos & 1 & 2 & 3 \\
\hline V38 & Partidos o grupos políticos & 1 & 2 & 3 \\
\hline V39 & $\begin{array}{l}\text { Asociaciones de conservación, medioambientales, } \\
\text { ecológicas y de derechos de los animales }\end{array}$ & 1 & 2 & 3 \\
\hline V40 & Asociaciones profesionales & 1 & 2 & 3 \\
\hline V41 & $\begin{array}{l}\text { Las diversas ONG (servicios sociales, salud, } \\
\text { derechos humanos, humanitarias) }\end{array}$ & 1 & 2 & 3 \\
\hline V42 & Otras (anotar): & 1 & 2 & 3 \\
\hline
\end{tabular}

Fuente: encuesta sobre desigualdad y capital social en España (2011).

plantea un amplio conjunto de cuestiones relativas a las distintas expresiones del capital social individual, esto es, a las redes de relaciones de que dispone un individuo y, entre ellas, la pertenencia a los diversos tipos de asociacionismo (Yang, 2007). Concretamente, la pregunta fue planteada en los términos que se pueden ver en la tabla $1^{9}$.

Un primer análisis descriptivo nos permitirá aproximar tanto los niveles de participación asociativa actuales como la configuración de dicha participación. En el gráfico 1 se observan los niveles de pertenencia derivados de la encuesta. Uno de los aspectos más destacables de estos resultados es el predominio de las ONG, que, en la sociedad española, integran ya a un 18,6\% de la población

nivel de confianza del $95,5 \%(z=2)$ y suponiendo $\mathrm{P}=\mathrm{Q}=0,5$. El trabajo de campo tuvo lugar entre noviembre y diciembre de 2011 mediante entrevista telefónica asistida por ordenador (Sistema CATI). La selección de hogares se hizo al azar. Se realizó a partir de listados telefónicos con selección fortuita de entrevistados por tablas aleatorias entre los miembros de cada hogar. Se optó por muestreo estratificado con afijación mixta: 1700 entrevistas por afijación simple para garantizar un mínimo de 100 entrevistas por comunidad autónoma y 1700 entrevistas por afijación proporcional al tamaño de cada comunidad autónoma. En cada una de ellas se realizó un reparto proporcional de las entrevistas por tamaño de municipio. Igualmente, se realizó una ponderación de los cuestionarios por sexo, edad y tamaño de cada comunidad autónoma. El cuestionario se encuentra disponible en la página web del grupo OSIM: http://osimudc.es/

9. En la encuesta se opta por una tipología del movimiento asociativo en ocho grupos siguiendo el esquema propuesto en la Encuesta Mundial de Valores. Este es casi idéntico a la Encuesta Europea de Valores, de forma que puede considerarse la clasificación más generalizada en su uso (Vázquez García, 2010). Además de su generalidad, esta tipología no adopta una postura teórica concreta y permite realizar múltiples agrupaciones de nivel superior en razón de los criterios adoptados. 
Gráfico 1. Porcentaje de pertenencia a asociaciones distinguiendo participación de participación activa

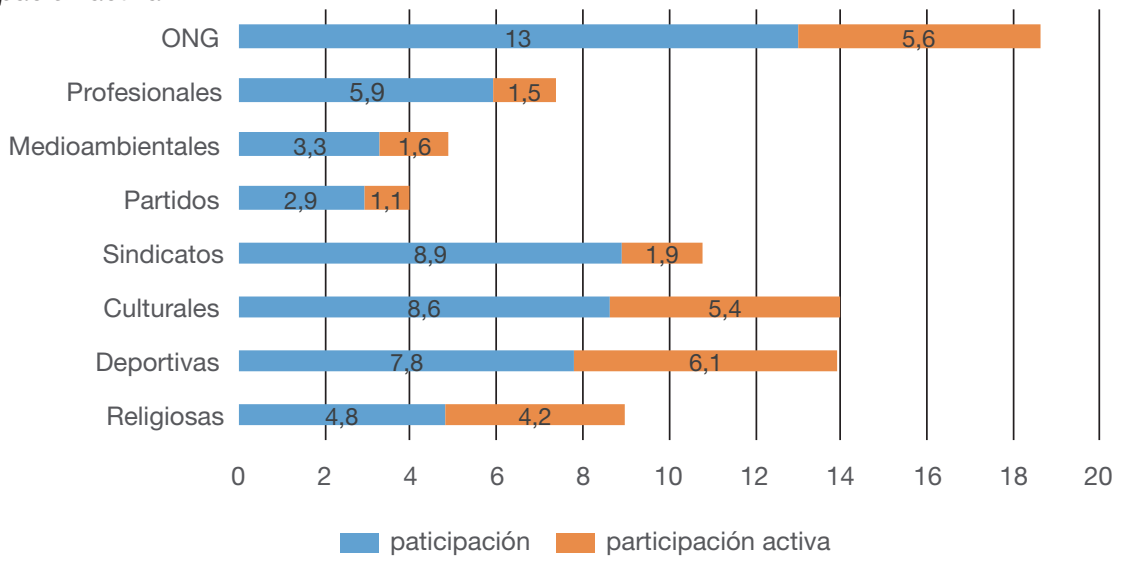

Fuente: elaboración propia a partir de la encuesta sobre capital social y desigualdad en España (2011).

mayor de 16 años, dato este que las situaría como el eje articulador del movimiento asociativo (Ariño, 2004). En el extremo opuesto se encuentra la escasa integración en el asociacionismo de corte político, un 4\%. Los datos que reflejaba la Encuesta Mundial de Valores ${ }^{10}$ de 1995 señalaban un nivel de pertenencia a organizaciones religiosas próximo al $40 \%$, a sindicatos por encima del $10 \%$ y a partidos en torno al $8 \%$. Las organizaciones culturales y deportivas presentaban porcentajes equivalentes o muy próximos. Frente a estas, las ONG y las organizaciones humanitarias en general estaban por debajo del 10\%. En contraste, de acuerdo con los datos más actuales, el asociacionismo de corte religioso muestra un nivel notablemente menor y se incrementa el peso de expresiones propias de una sociedad secular (las asociaciones deportivas, las culturales y las ONG). En lo que respecta al asociacionismo político, su evolución descendente se viene evidenciando desde finales de los años setenta, lo que parece revelar un constante proceso de desideologización del movimiento asociativo (Alberich, 2007).

En lo relativo a la evolución de la pertenencia asociativa, existe un amplio conjunto de estudios sobre el caso del asociacionismo en España (Marban, 2003; De la Torre, 2005; Pérez-Díaz, 2002; Ruiz Olabuénaga, 2005; Subirats, 1999). La amplia variabilidad de resultados derivada de problemas metodológicos (Ariño, 2007) hace extremadamente difícil reconstruir una serie temporal. Siguiendo un criterio de similitud metodológica, establecemos una comparación con las cifras que arrojan las encuestas del $\mathrm{CIS}^{11}$. En este sentido, cabe destacar que en el período que va desde 1981, en el que se parte de cifras de pertenencia del $18 \%$, se ha producido un crecimiento casi continuado hasta

10. La Encuesta Mundial de Valores (EMV) presenta el mismo sistema de clasificación que las organizaciones.

11. CIS, encuestas de ciudadanía y participación 2450, 2575 y 2632. 
Gráfico 2. Porcentaje de participación activa según tipología asociativa

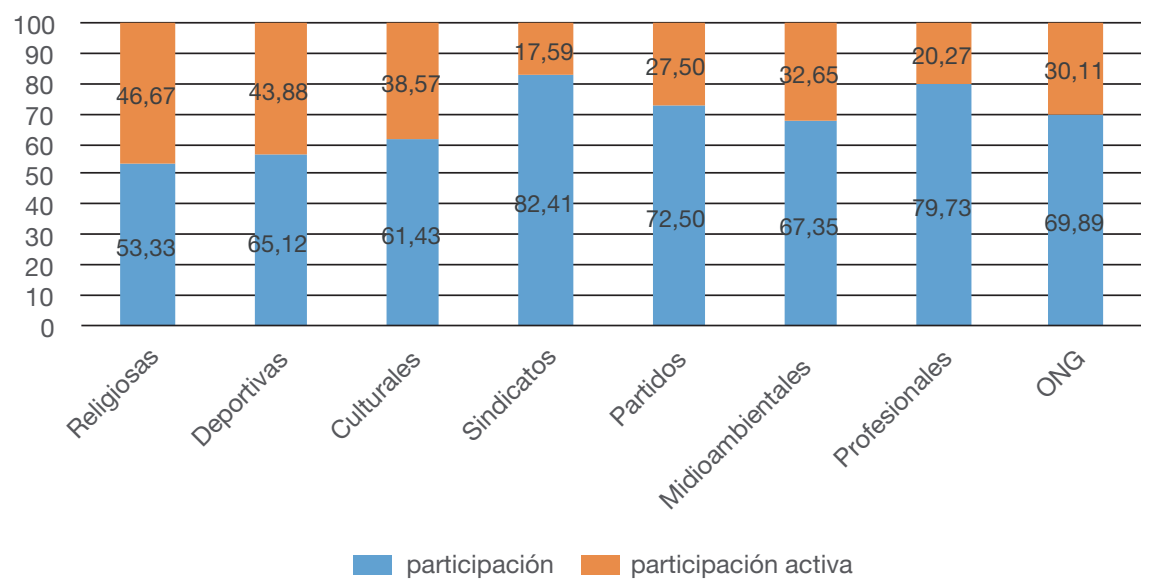

Fuente: elaboración propia a partir de la encuesta sobre capital social y desigualdad en España (2011).

el 47,3\% que refleja nuestra encuesta. Igualmente, la participación múltiple ha experimentado un fuerte crecimiento desde el $4 \%$ en 1981 hasta el $20 \%$ en 2011. Ambos resultados son coherentes con la hipótesis de la modernización según la cual se ha producido una ampliación de la pertenencia y del crecimiento de la multiafiliación, unida a una transformación en las formas de participación social (Ariño, 2006; Morales, 2003; Morales y Mota, 2006; Marcuello, 2007; Putnam, 2000; Norris, 2002).

Los resultados previos suponen exclusivamente la valoración de una pertenencia a los distintos tipos de asociaciones. Los datos sobre participación activa en las mismas exige realizar algunas puntualizaciones (véase el gráfico 2). La participación activa frente a la mera adscripción alcanza el 46,67\% en las organizaciones religiosas, el nivel más elevado. Por el contrario, la participación en sindicatos (un 17,59\%) y partidos políticos (un 27,5\%) resulta sensiblemente inferior. Esto es, en el caso de las asociaciones religiosas, la adscripción social se ha reducido sustancialmente como consecuencia lógica del proceso de secularización ${ }^{12}$, pero la vinculación de los adscritos es notablemente superior frente a otras expresiones tradicionales. En general, se hace patente una escasa participación activa en un amplio conjunto de asociaciones o la extensión de la denominada "participación de talonario» (checkbook participants).

Ahora bien, esta información es parcial en la medida en que todavía no se consideran las múltiples pertenencias, es decir, el hecho de que individuos se integren en diversos sistemas asociativos interconectando grupos. A este respecto, el primer dato remarcable es que más de la mitad de la población no está integrada en ninguna forma de asociación. Del grupo restante, solo un 20,3\%

12. En particular, si tenemos en cuenta que tradicionalmente esta adscripción era parte de la identidad social en España. 
Gráfico 3. Frecuencia de pertenencia a múltiples asociaciones

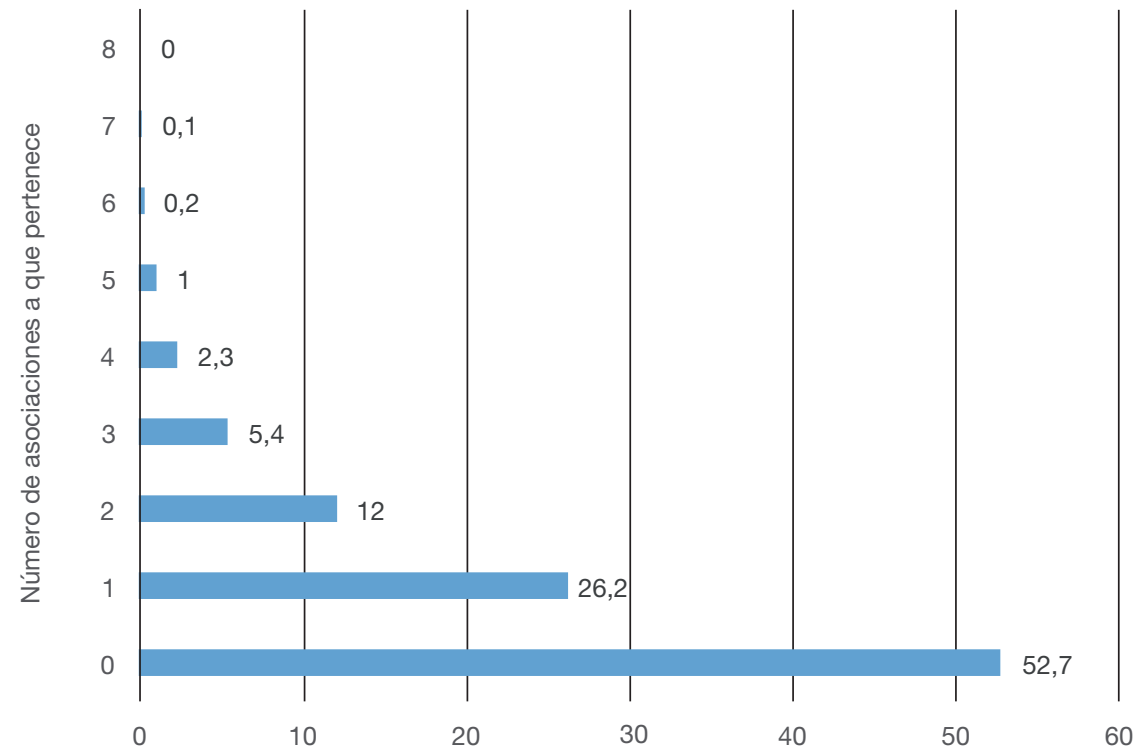

Fuente: elaboración propia a partir de la encuesta sobre capital social y desigualdad en España (2011).

de la población se integra en más de una asociación (véase el gráfico 3). Estas interrelaciones de pertenencias definen el conjunto más activo de ciudadanos interconectados por pertenencias múltiples.

Un simple análisis de las correlaciones existentes entre las respuestas a la participación en las diversas expresiones asociativas (tabla 2) pone de relieve la pérdida del carácter central que en su momento ocupó el asociacionismo religioso y la creciente importancia del asociacionismo cultural y de las ONG. Estas últimas presentan correlaciones más altas con cualquier forma asociativa.

Tabla 2. Correlaciones de Pearson entre las pertenencias a asociaciones

\begin{tabular}{|c|c|c|c|c|c|c|c|}
\hline & Religiosas & Deportivas & Sindicatos & Partidos & Medioambientales & Profesionales & ONG \\
\hline Deportivas & $0,072^{\star \star}$ & & & & & & \\
\hline Sindicatos & 0,009 & $0,075^{\star \star}$ & & & & & \\
\hline Partidos & 0,016 & $0,085^{\star \star}$ & $0,272^{\star \star}$ & & & & \\
\hline Medioambientales & 0,023 & $0,093^{\star \star}$ & $0,108^{\star \star}$ & $0,150^{\star \star}$ & & & \\
\hline Profesionales & $0,037^{\star}$ & $0,170^{\star \star}$ & $0,119^{\star \star}$ & $0,113^{\star \star}$ & $0,196^{\star \star}$ & & \\
\hline ONG & $0,100^{\star \star}$ & $0,102^{\star \star}$ & $0,094^{\star \star}$ & $0,113^{\star \star}$ & $0,199^{\star \star}$ & $0,119^{\star *}$ & \\
\hline Culturales & $0,104^{\star \star}$ & $0,160^{\star \star}$ & $0,112^{\star \star}$ & $0,164^{\star \star}$ & $0,213^{\star \star}$ & $0,195^{\star \star}$ & $0,178^{\star \star}$ \\
\hline
\end{tabular}

* La correlación es significante al nivel 0,05 (bilateral).

** La correlación es significativa al nivel 0,01 (bilateral).

Fuente: elaboración propia a partir de la encuesta sobre capital social y desigualdad en España (2011). 
En este sentido, dado que buscamos determinar la existencia de factores subyacentes en la pertenencia, hemos optado por utilizar una técnica de reducción de datos: el análisis de componentes principales. La aplicación de dicho método a este caso se encuentra con el problema de que se trata de variables no continuas binomializadas. Esta particularidad conlleva la necesidad de tomar como punto de partida las correlaciones tetracóricas existentes entre cada una de las variables integradas en la batería de preguntas (Freiberg et al., 2013) para construir los factores.

De este modo reducimos las pertenencias a múltiples formas de asociacionismo a un menor conjunto de variables (en este caso, dos) que recogen tanto la integración en múltiples asociaciones como las interrelaciones existentes entre las mismas. Por una parte, se encuentra la dimensión secular, donde puntúa fuertemente la pertenencia a sindicatos, partidos políticos o asociaciones profesionales, en general instituciones centradas en la defensa de un interés concreto. Frente a estas, la integración en el asociacionismo religioso coincide con la forma más tradicional de asociacionismo en España. En una posición intermedia se sitúan las asociaciones deportivas, las culturales y las ONG. Ahora bien, las cargas factoriales de estas últimas, aunque manifiestan peso en ambos factores, son superiores en el eje que hemos denominado «secular». Es decir, las interrelaciones entre las diversas formas de asociacionismo son más fuertes entre las emergentes y las formas políticas que entre estas y las religiosas. De hecho, estas últimas comienzan a mostrarse más aisladas de las expresiones restantes.

Trabajos previos han constatado la existencia de dos tríadas de redes asociativas para la realidad social española, cada una de las cuales se vincula a un modelo social: el mundo social de la Iglesia y su relación con la caridad y la asistencia social; el eje formado por Iglesia, cultura y deporte, y un modelo que podría calificarse como más moderno ejemplificado por el eje formado por sindicatos, cultura y deporte (Rodríguez et al., 2003). Aunque es aventurado afirmar que se constata una evolución en un sentido determinado, las evidencias derivadas de nuestro estudio ponen de relieve que existen cambios en las estructuras asociativas tradicionales que ya habían sido patentes en otros trabajos (Vázquez, 2010). En concreto, pese a que la Iglesia todavía representa un papel relevante en la sociedad civil española, su espacio centralizador de acción ha sido ocupado por otras instituciones civiles, en particular las ONG y las organizaciones culturales, y ello a pesar del desarrollo del estado benefactor y de la envergadura de la acción asistencial pública. Estas organizaciones configuran un espacio propio con un alto nivel participativo, incluida la participación activa, esto es, se trata de un asociacionismo de corte horizontal. En este caso es importante notar el alto nivel de relación entre estas expresiones asociativas y su incremento constante en las últimas décadas. El peso del asociacionismo religioso, ligado al cultural, a las ONG, etc., junto con el ideológico o instrumental, ha dejado paso al predominio de un asociacionismo expresivo pero desvinculado tanto de lo religioso como del más ideologizado (Rodríguez et al., 2003; Vázquez, 2010). 
Gráfico 4. Cargas factoriales de las pertenencias asociativas (rotación varimax)

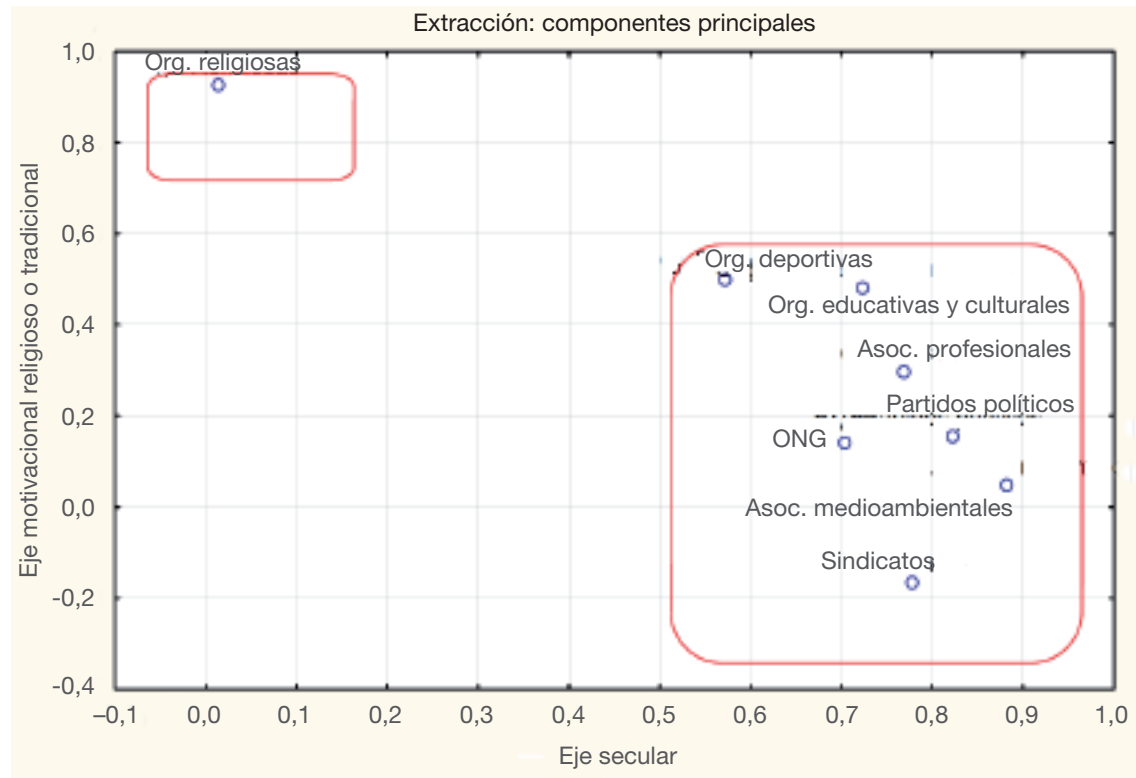

Fuente: elaboración propia a partir de la encuesta sobre capital social y desigualdad en España (2011).

\subsection{Asociacionismo y confianza general en la sociedad española}

Las dimensiones derivadas del Análisis de Componentes Principales (ACP) recogen los niveles de participación de cada individuo en los modelos asociativos anteriormente mencionados, de forma que pueden denominarse «dimensión asociacionismo religioso y secular». El primero de ellos presenta una muy baja integración en otros movimientos asociativos, frente al segundo que es de carácter abierto. Las correlaciones de Pearson (tabla 3) entre las dimensiones derivadas del ACP, los diversos indicadores de capital social y las variables sociodemográficas ponen de manifiesto una particular relación entre asociacionismo y la confianza generalizada ${ }^{13}$ acorde con la hipótesis de Paxton. En particular, los resultados revelan que no todas las formas de asociacionismo inciden del mismo modo en la generación de capital social. Concretamente, lo que hemos denominado «asociacionismo religioso" muestra una relación divergente con gran parte de las variables consideradas. A su vez, de la propia lógica del análisis de componentes prin-

13. La medición de la confianza se realiza mediante una pregunta estandarizada en la Encuesta Mundial de Valores: «En términos generales, ¿cree usted que es posible confiar en la mayor parte de la gente o que nunca se es demasiado cuidadoso en el trato con la gente?». Este interrogante ha venido siendo respondido durante cuatro décadas en un amplio conjunto de países. La cuestión que plantea no está exenta de controversias (Newton, 2001), aunque se ha convertido en el indicador proxy de capital social generalmente aceptado. 
Tabla 3. Correlaciones de los indicadores del capital social individual

\begin{tabular}{|c|c|c|}
\hline & $\begin{array}{l}\text { Asociacionismo } \\
\text { secular }\end{array}$ & $\begin{array}{l}\text { Asociacionismo } \\
\text { religioso tradicional }\end{array}$ \\
\hline Tamaño del municipio & 0,021 & 0,022 \\
\hline Tiempo de residencia & $-0,131^{\star \star}$ & 0,033 \\
\hline Confianza generalizada & $0,131^{\star *}$ & 0,040 \\
\hline Satisfacción vital & $0,050^{\star *}$ & $0,064^{* *}$ \\
\hline Salud física & $0,056^{\star *}$ & 0,023 \\
\hline Salud mental & $0,046^{\star *}$ & $0,067^{\star *}$ \\
\hline Seguridad residencial percibida & $0,072^{\star \star}$ & $-0,002$ \\
\hline Felicidad & $0,058^{\star \star}$ & $0,049^{\star \star}$ \\
\hline Actividad & $0,215^{\star *}$ & $-0,058^{\star \star}$ \\
\hline Edad & $-0,085^{\star \star}$ & $0,084^{\star *}$ \\
\hline Sexo & $0,106^{* *}$ & $-0,013$ \\
\hline Estado civil & $0,079^{* *}$ & 0,003 \\
\hline Número hogar & $0,068^{\star *}$ & $-0,024$ \\
\hline Nivel de estudios & $0,247^{\star \star}$ & $0,050^{* *}$ \\
\hline Ingresos & $0,250^{\star *}$ & $0,050^{*}$ \\
\hline Asociacionismo secular & 1 & $-0,155^{\star \star}$ \\
\hline Asociacionismo religioso & $-0,155^{\star \star}$ & 1 \\
\hline
\end{tabular}

cipales, se desprende que este tipo de asociacionismo guarda una relación inversa con las restantes formas de asociacionismo y una escasa relación con la confianza generalizada o la percepción de seguridad en el entorno. Este hecho puede ser explicado por la reconfiguración del sistema asociativo o su secularización progresiva. Estas formas asociativas antes predominantes se han ido aislando progresivamente de las restantes expresiones asociativas, envejecidas y ligadas a grupos sociales que presentan poca movilidad social y geográfica.

Por el contrario, el asociacionismo secular muestra correlaciones positivas con todas las expresiones macro del capital social (confianza y seguridad residencial percibida). Esta variable responde a la lógica de los procesos inversores (Glaeser et al., 2002), disminuye con la edad y el sexo (Muñoz, 2013) y se eleva con el nivel de ingresos y de educación. Una particularidad, aparentemente contraintuitiva, es que la estabilidad residencial muestra una relación inversa con la extensión de la red personal. Este hecho puede ser explicado por la mayor movilidad que presentan los individuos con un estatus y un capital social más elevados.

Con el objeto de profundizar en el análisis descriptivo, llevamos a cabo un análisis de regresión logística binaria (tabla 4) que nos permitirá identificar los factores potencialmente explicativos de la confianza generalizada. Para ello, junto a los factores derivados del ACP, se introdujeron variables sociodemo- 
Tabla 4. Determinantes de la confianza generalizada (logit binomial, $n=3400$ )

\begin{tabular}{|c|c|c|c|}
\hline & Modelo I & Modelo II & Modelo III \\
\hline Constante & $-3,66055^{\star \star \star}$ & $-3,271^{\star \star \star}$ & $-3,2251^{\star \star *}$ \\
\hline Tamaño del municipio & $-0,0122$ & & \\
\hline Tiempo de residencia & 0,0016 & & \\
\hline Satisfacción vital & $0,1041^{* \star *}$ & $0,1428^{\star \star \star}$ & $0,1416^{\star \star *}$ \\
\hline Salud física & 0,0307 & & \\
\hline Salud mental & 0,0024 & & \\
\hline Felicidad & 0,1319 & & \\
\hline Actividad & 0,0547 & 0,0840 & \\
\hline Edad & 0,0866 & 0,0271 & $0,1274^{\star \star \star}$ \\
\hline Edad 2 & 0,0071 & 0,0150 & \\
\hline Sexo & 0,0592 & 0,0521 & \\
\hline Nacionalidad & 0,0980 & 0,1229 & \\
\hline Hijos & 0,0051 & 0,0063 & \\
\hline Estado civil & $-0,1977^{\star}$ & $-0,1882^{\star}$ & $-0,1903^{\star}$ \\
\hline Número de miembros del hogar & $-0,0089$ & & \\
\hline Ingresos del hogar & $0,0934^{\star \star \star}$ & $0,0845^{\star \star \star}$ & $0,0974^{\star \star \star}$ \\
\hline Nivel de estudios & $0,2236^{\star \star \star}$ & $0,2261^{\star \star \star}$ & $0,2269^{\star \star \star}$ \\
\hline Asociacionismo secular & $0,5216^{\star \star \star}$ & $0,5183^{\star \star \star}$ & $0,5366^{\star \star \star}$ \\
\hline Asociacionismo religioso & 0,1415 & 0,1537 & 0,1479 \\
\hline Pseudo $\mathrm{R}^{2^{2+x+k}}$ McFadden & 0,3981 & 0,3906 & 0,3900 \\
\hline Pseudo $\mathrm{R}^{2}$ corregido & 0,3907 & 0,3856 & 0,3860 \\
\hline Log-verosimilitud & $-1396,5$ & $-1414,052$ & $-1415,213$ \\
\hline Predicción correcta & $62,3 \%$ & $62 \%$ & $61,4 \%$ \\
\hline
\end{tabular}

* Significativo al 0,1 .

** Significativo al 0,05 .

*** Significativo al 0,01 .

* Como es obvio, este resultado no es interpretable como porcentaje de la varianza explicada, sino como mejoría relativa respecto a un modelo naíf en el que sólo figurase el término independiente. Esto es, se trata de modelos con capacidad explicativa relevante.

Fuente: elaboración propia a partir de los datos de la encuesta sobre desigualdad y capital social en España (2011).

gráficas y de entorno, así como variables de control que permitirían incorporar al análisis el papel de la predisposición psicológica a la confianza.

Los resultados de nuestras estimaciones confirman lo observado en el análisis de las correlaciones y evidencian la capacidad explicativa que presenta el asociacionismo abierto sobre la confianza generalizada. En este sentido, los resultados ofrecen evidencia favorable a lo propuesto por la hipótesis de Paxton. Ahora bien, igualmente reconocen capacidad explicativa a la propuesta de Uslaner, en tanto que sería sostenible que la confianza fuera también función del bienestar psicológico. De hecho, la confianza generalizada muestra una relación significativa con la satisfacción vital y con variables como el nivel de 
estudios e ingresos, esto es, con las que están ligadas al control sobre el contexto. No obstante, esto solo supone la existencia de una interrelación entre las variables mencionadas, dado el problema de endogeneidad entre confianza y satisfacción vital.

En el modelo I optamos por introducir un amplio conjunto de variables potencialmente explicativas, eliminando aquellas con las que existe una fuerte identificación o relaciones endógenas (probabilidad de comportamiento oportunista o percepción de seguridad residencial). Sobre los resultados solo serían explicativas la satisfacción vital, el nivel de ingresos y de estudios y la participación en asociaciones abiertas. Dadas la potenciales multicolinealidades, en el modelo II hemos eliminado aquellas variables que presentaban muy baja significatividad. Los resultados son totalmente estables. En conjunto, la proxy del capital social (la confianza generalizada) se encuentra relacionada con factores de estatus como el nivel de ingresos o de estudios. Esto es, por variables ligadas al control del entorno social. Junto a ello, con un cierto componente endógeno, la satisfacción vital presenta una relación altamente significativa. Finalmente, tal y como predice la hipótesis de Paxton, no todas las formas de asociacionismo influyen en el capital social, sino exclusivamente aquellas que pueden calificarse como abiertas por su interrelación con otras.

En esta misma línea, cabe una reinterpretación de los resultados en razón de la denominada "controversia Olson-Putnam». Al igual que en la propuesta de Paxton, desde la perspectiva de Olson (1982), no todas las expresiones asociativas son favorecedoras de la generación de confianza generalizada, sino que grupos cerrados a la captura de intereses particulares pueden tener un carácter deletéreo del funcionamiento social. En trabajos previos (Pena y Sánchez, 2007) ya se había puesto de manifiesto que el asociacionismo de carácter horizontal presentaba rasgos de bien público frente a otras expresiones más verticales. Dicho de otro modo, el mismo resultado de interacción entre asociaciones puede ser reinterpretado en términos de la naturaleza de las asociaciones. En nuestra muestra este carácter de bien público es atribuible en la actualidad a las ONG y a las asociaciones culturales.

\section{Conclusiones}

Investigaciones previas han venido insistiendo en la vinculación del asociacionismo con la confianza mediante argumentos que inevitablemente son circulares, de forma que se establece una identidad no contrastada entre confianza y asociacionismo en cualquiera de sus formas y de su capital social. En este sentido, se contrasta en qué medida la pertenencia asociativa, en particular las múltiples pertenencias asociativas que propone Paxton, son determinantes de la confianza generalizada. Para esta hipótesis, solo el asociacionismo abierto que permite la interconexión de grupos es generador de confianza generalizada.

El análisis de los resultados de la encuesta realizada en España (OSIM, 2011) nos permite tanto contrastar la evolución del asociacionismo y de la participación en España como las interrelaciones entre las pertenencias asociativas. 
Sobre los datos se aprecia tanto un sensible declive como una reestructuración. Así, se observa la extensión de formas de asociacionismo horizontal y abierto frente a la reducción del peso del asociacionismo tradicional. Los indicadores ligados al asociacionismo, igualmente, muestran una clara estructura bidimensional dependiente de la naturaleza de las organizaciones y que refleja una transición en los modelos asociativos.

En el estudio de la causalidad para el caso español, no todas las redes sociales son equiparables en la generación de capital social. Las relaciones funcionales observadas entre el principal indicador de capital social, la confianza generalizada y las dimensiones observadas apoyan la hipótesis de Paxton (2007) sobre la influencia de la integración en formas de asociacionismo abierto o interrelacionado con otras expresiones asociativas. Estas configuran una red amplia que pone en contacto (establece puentes) entre individuos de grupos diversos, transformando la confianza particular en percepción de confianza general. Para esta propuesta, el capital social no se deriva del asociacionismo en todas sus expresiones, sino exclusivamente aquel que puede calificarse como abierto por su interrelación con otros. En conjunto, la percepción de confianza general o de ausencia de comportamientos oportunistas se encuentra más relacionada con la educación y el acceso y la movilización de una red de lazos facilitada por la pertenencia a asociaciones abiertas que facilitan puentes entre grupos diversos. Este resultado fue obtenido controlando la influencia de otras variables que se han venido mostrando significativas, como el bienestar psicológico y la experiencia de seguridad y de control sobre el entorno (Uslaner, 2002). En este sentido, tal y como señalamos, no se trata de propuestas incompatibles. De un lado, es evidente que la experiencia de control sobre el medio que traduce la satisfacción vital está ligada a la confianza general, pero también la existencia de una activa interacción asociativa y la multiplicación de los contactos sociales ejercen una influencia directa sobre la misma.

El trabajar con datos de sección cruzada supone una limitación a la hora de establecer conclusiones sobre la dirección de causalidad para la contrastación de la hipótesis de Paxton. La existencia de este tipo de limitaciones orienta las futuras líneas de investigación que permitan superarlas. Del mismo modo, el carácter central de las ONG y de las asociaciones culturales introduce la posibilidad de extender el análisis a la naturaleza de las asociaciones generadoras de confianza como bienes públicos, tal y como propone Sonderskov (2011).

\section{Referencias bibliográficas}

Adler, P.A. y KWOn, S.W. (2002). «Social capital: Prospects for a new concept». Academy of Management Review, 27 (1), 5-40. $<$ https://doi.org/10.2307/4134367>

Alberich, T. (2007). «Contradicciones y evolución de los movimientos sociales en España». Documentación Social, 145, 183-210.

AriÑo, A. (2004). «Asociacionismo, ciudadanía y bienestar social». Papers, 74, 85-110. <https://doi.org/10.5565/rev/papers/v74n0.1088> 
- (2007). Asociacionismo y voluntariado en España: Una perspectiva general. Valencia: Tirant lo Blanch.

Bacharach, M. y GambetTa, D. (2001). «Trust in signs». En: CoOK, K. (ed.). Trust in Society. Nueva York: Russell Sage Foundation.

BARBER, B. (1983): The Logic and Limits of Trust. New Brunswick: Rutgers University Press.

BECKER, L. (1996). "Trust as non-cognitive security about motives». Ethics. 107, 43-61. <https://doi.org/10.1086/233696>

Bourdieu, P. (2001). "The forms of capital». En: GranovetTer, M. y SwedberG, R. The sociology of economic life. 2. ${ }^{\text {a }}$ ed. Oxford: Westview Press, 97-111. <https://doi.org/10.1002/9780470755679.ch15>

BOWLES, H. y GINTIS, S. (2002). «Social capital and community governance». Economic Journal, 112 (483), 419-436.

Burns, N. y Kinder, D. (2000). Social Trust and Democratic Politics. Report to the National Election Studies Board. Based on the 2000 NES Special Topic Pilot Study.

Claibourn, M.P. y Martin, P. (2000). «Trusting and Joining: An Empirical Test of the Reciprocal Nature of Social Capital». Political Behavior, 22, 267-291.

Coleman, J. (1988). "Social capital in the creation of human capital». American Journal of Sociology, 94, 95-120. <https://doi.org/10.1016/b978-0-7506-7222-1.50005-2>

- (1990). Foundations of Social Theory. Cambridge, Mass.: Harvard University Press.

Dasgupta, P. y Serageldin, I. (2001). Social capital: A multifaceted approach. Washington: World Bank. $<$ https://doi.org/10.1596/0-8213-4562-1>

Delhey, J. y NewTon, K. (2005). «Predicting Cross-National Levels of Social Trust: Global Pattern or Nordic Exceptionalism?». European Sociological Review, 21 (4), 311-327. <https://doi.org/10.1093/esr/jci022>

DelheY, J.; NEWTON, K. y WelzEL, C. (2011). «How is general trust in "most people”?: Solving the radius trust problem». American Sociological Review, 76, 786-807. <https://doi.org/10.1177/0003122411420817>

EtzIOnI, A. (2007). La dimensión moral: Hacia una nueva economía. Madrid: Ediciones Palabra.

FreiberG, A.; Stover, J. e Iglesia, G. de la (2013). «Correlaciones policóricas y tetracóricas en estudios factoriales». Ciencias Psicológicas, VII (2), 151-164.

Glaeser, E.; Laibson, D. y SACERDote, B. (2002). «An economic approach to social capital». Economic Journal, 112, 437-458. <https://doi.org/10.1111/1468-0297.00078>

Glanville, J.L. y Paxton, P. (2007). «How Do We Learn to Trust?: A Confirmatory Tetrad Analysis of the Sources of Generalized Trust». Social Psychology Quarterly, 70, 230-242. <https://doi.org/10.1177/019027250707000303>

GranovetTer, M. (1973). "The strength of weak ties». American Journal of Sociology, 78, 1360-1380. <https://doi.org/10.1086/225469>

Hardin, R. (2001). "Conceptions and Explanations of Trust». En: CoOK, K.S. (ed.). Trust in Society. 1. a ed. Vol. 2. Nueva York: Russell Sage Foundation, 3-39. The Russell Sage Foundation Series on Trust. 
- (2002). Trust and Trustworthiness. Londres: Russell Sage Foundation.

Hearn, F. (1997). Moral Order and Social Disorder: The American Search for Civil Society. Nueva York: Aldine de Gruyter. <https://doi.org/10.5860/choice.35-3601>

Herreros VÁzQuez, F. (2002). ¿Por qué confiar?: El problema de la creación del capital social. Madrid: Centro de Estudios Avanzados en Ciencias Sociales. Instituto Juan March.

- (2003). «Las fuentes de la confianza social». Revista Internacional de Sociología, 35, 151-175.

<https://doi.org/10.3989/ris.2003.i35.304>

- (2008). "La confianza y la cooperación en la ausencia de Estado». Revista Internacional de Sociología, 46, 87-105.

HoGG, M.A. (1992). The social psychology of group cohesiveness: From attraction to social identity. Nueva York: New York University Press.

Jones, K. (1996). «Trust as an Affective Attitude». Ethics, 107, 4-25. <https://doi.org/10.1086/233694>

Luhmann, N. (1988). «Familiarity, Confidence, Trust: Problems and Alternatives». En: Gambetta, D. (ed.). Trust: Making and Breaking of Cooperative Relations. Oxford: Blackwell, 94-107.

Marban GALlEgO, V. (2003). «Actitudes y valores sobre la participación en la sociedad civil en España y Europa». En: RodríGuez Cabrero, G. (coord.). Las entidades voluntarias de acción social en España. Madrid: Fundación Foessa.

MarCuello, C. (2007). Capital social y organizaciones no lucrativas en España: El caso de las ONGD. Madrid: Fundación BBVA.

Marsden, P.V. y FriedKIN, N.E. (1993). «Network studies of social influence». Sociological Methods \& Research, 22 (1), 127-151. <https://doi.org/10.1177/0049124193022001006>

Millán, R. y Gordon, S. (2004). "Capital social: Una lectura de tres perspectivas clásicas». Revista Mexicana de Sociología, 66 (4), 711-746. <https://doi.org/10.2307/3541414>

Moody, J. y White, D. (2003). «Structural Cohesion and Embeddedness: A Hierarchical Concept of Social Groups». American Sociological Review, 68 (1), 103-127. <https://doi.org/10.2307/3088904>

Morales, L. (2003). Ever Less Engaged Citizens?: Political Participation and Associational Membership in Spain. Barcelona: Institut de Ciències Polítiques i Socials. Working Papers, 220.

- (2006). Instituciones, movilización y participación política: El asociacionismo político en las democracias occidentales. Madrid: Centro de Estudios Políticos y Constitucionales.

Morales, L. y Mota, F. (2006). «El asociacionismo en España». En: MonTero, J.R.; FOnT, J. y TORCAL, M. Ciudadanos, asociaciones y participación en España. Madrid: CIS, 77-104.

Muñoz Goy, C. (2013). «Social capital in Spain: Are there gender differences?». European Journal of Government and Economics, 2 (1), 79-94.

Newton, K. (2001). "Trust, Social Capital, Civil Society, and Democracy». International Political Science Review, 22 (2), 201-214. <https://doi.org/10.1177/0192512101222004>

Norris, P. (2002). Democratic Phoenix: Reinventing Political Activism. Cambridge: Cambridge University Press. 
Olson, M. (1982). The Rise and Decline of Nations: Economic Growth, Stagnation and Social Rigidities. Yale: Yale University Press.

OSIM, (2011). Encuesta sobre desigualdad y capital social, disponible en: <http:// osimudc.es>

Ostrom, E. y Walker, J. (comps.) (2003). Trust and Reciprocity: Interdisciplinary Lessons from Experimental Research. Nueva York: Russell Sage Foundation.

PALDAM, M. (2000). «Social capital: one or many?: Definition and measurement». Journal of Economic Surveys, 14 (5), 629-653. <https://doi.org/10.1111/1467-6419.00127>

Paxton, P. (1999). «Is Social Capital Declining in the United States?: A Multiple Indicator Assessment». American Journal of Sociology, 105 (1), 88-127. <https://doi.org/10.1086/210268>

- (2002). «Social Capital and Democracy: An Interdependent Relationship». American Sociological Review, 67, 254-277. <https://doi.org/10.2307/3088895>

- (2007). «Association Memberships and Generalized Trust: A Multilevel Model across 31 Countries». Social Forces, 86, 47-76. <https://doi.org/10.1353/sof.2007.0107>

PENA LóPEZ, J.A. y SÁnCHEZ SANTOS, J.M. (2005). «Actividad asociativa, confianza y generación de capital social: Evidencia empírica». Ekonomiaz, 59, 137-159.

- (2007). «The Olson-Putnam controversy: Some empirical evidence». Economics Bulletin, 26 (4), 1-10.

- (2014). «Does Corruption Have Social Roots?: The Role of Culture and Social Capital». Journal of Business Ethics, 12 (4), 697-708. <https://doi.org/10.1007/s10551-013-1789-9>

PÉrEZ-DíAZ, Víctor (2002). «From Civil War to Civil Society: Social Capital in Spain 1930s/1990s». En: Putnam, R. (ed.). Democracies in Flux. Nueva York: Oxford University Press. <https://doi.org/10.1093/0195150899.003.0007>

PORTES, A. (2000). «The two meanings of social capital». Sociological Forum, 15 (1), 1-12.

Putnam, R. (1993). Making democracy work: Civic traditions in modern Italy. Princeton: Princeton University Press.

- (1995). «Bowling alone: America's declining social capital». Journal of Democracy, 6, 65-78.

$<$ https://doi.org/10.1353/jod.1995.0002>

- (2000). Bowling alone: The collapse and revival of American community. Nueva York: Simon and Schuster. <https://doi.org/10.1007/978-3-658-13213-2_95>

RodrígueZ, J.A.; BOSCH, J. y MÉRIDA, F. (2003). El capital social como vertebrador de la sociedad. Working Paper. Universidad de Barcelona.

Ruiz OlabuÉNAGA, J. (2005). «El tercer sector español y sus campos de actuación». Revista Electrónica del Tercer Sector, 1. Fundación Luis Vives.

Sobel, J. (2002). "Can we trust social capital?». Journal of Economic Literature, 40 (1), 139-154. <https://doi.org/10.1257/jel.40.1.139>

Sonderskov, K.M. (2011). «Does Generalized Social Trust Lead to Associational Membership?: Unraveling a Bowl of Well-Tossed Spaghetti». European Sociological Review, 27 (4), 419-434. <https://doi.org/10.1093/esr/jcq017> 
Sorrentino, R.; Holmes, J.G.; STEVen, E. y SHARP, Ann (1995). «Uncertainty orientation and trust in close relationships: Individual differences in cognitive styles». Journal of Personality and Social Psychology, 68 (2), 314-327. <https://doi.org/10.1037/0022-3514.68.2.314>

STOlle, D. (2001). «Getting to Trust: An Analysis of the Importance of Institutions, Families, Personal Experiences and Group Membership». En: DeKKer, Paul y Uslaner, Eric M. Politics in Everyday Life: Social Capital and Participation. Londres: Routledge.

Stolle, D. y Rochon, T. (1998). "Are All Associations Alike?». En: Edwards, B. y Foley, M. (eds.). «Beyond Tocqueville: Civil Society and Social Capital in Comparative Perspective». American Behavioral Scientist, 42 (1), 47-65. <https://doi.org/10.1177/0002764298042001005>

Subirats, J. (ed.) (1999). ¿Existe sociedad civil en España?: Responsabilidades civiles y valores públicos. Madrid: Fundación Encuentro.

TORRE, Isabel de la (2005). Tercer sector y participación ciudadana en España. Madrid: CIS.

Uslaner, E.M. (1999). «Trust But Verify: Social Capital and Moral Behavior». Social Science Information, 38, 29-56. <https://doi.org/10.1177/053901899038001002>

- (2002). The Moral Foundations of Trust. Cambridge: Cambridge University Press.

- (2008). "Trust as a moral value». En: Castiglione, D.; Van Deth, J.W. y Wolleb, G. (eds.). The Handbook of Social Capital. Nueva York: Oxford University Press, 101-121.

VAN INGEN, Erik y BEKKERS. R. (2015). «Generalized trust through civic engagement?: Evidence form five national panel studies». Political Psychology, 36 (3), 277-294. <https://doi.org/10.1111/pops.12105>

VÁzQueZ García, Rafael (2010). Compromiso cívico y democracia: Los efectos democráticos del asociacionismo sociopolítico en España. Sevilla: Centro de Estudios Andaluces.

Williamson, O. (2000). «The New Institutional Economics: Taking Stock, Looking Ahead». Journal of Economic Literature, 38 (3), 595-613. <https://doi.org/10.1257/jel.38.3.595>

WinTER, I. (2001). Toward a Theorized Understanding of Family Life and Social Capital. Melbourne: Melbourne University. Working Paper, 21.

WoOlCOCK, M. (2001). "The place of social capital in understanding social and economic outcomes». Canadian Journal of Policy Research, 2 (1), 1492-1525.

YANG, K. (2007). «Individual social capital and its measurement in social surveys». Survey Research Methods, 1 (1), 7-27.

YosANO, A. y HAYASHI, Nahoko (2005). «Social Stratification, Intermediary Groups, and Creation of Trustfulness». Sociological Theory and Methods, 20, 27-44.

ZuCKER, L.G. (1986). «Production of Trust: Institutional Sources of Economic Structure, 1840-1920». Research in Organizational Behavior, 8, 53-111. 